\title{
Universal Nerve Conduction Screening in Type 1 Diabetes-Are We There Yet?
}

\author{
Anurag Bajpai ${ }^{1,2}$
}

Received: 21 December 2021 / Accepted: 27 December 2021 /Published online: 14 January 2022

c) Dr. K C Chaudhuri Foundation 2022

Type 1 diabetes represents one of the most common chronic disorders in children and adolescents. Timely identification and treatment of chronic complications is the primary goal of managing the condition. While there is a substantial body of evidence about the epidemiology and course of renal and retinal complications of type 1 diabetes; there is a paucity of information about neuropathy, a significant cause of longterm morbidity. Limited Indian data show a relatively low prevalence of clinical neuropathy in type 1 diabetes $[1,2]$. Understanding the time course and predictors of peripheral neuropathy is desirable in the Indian setting.

In this issue of the Journal, Singh et al. report the point prevalence and predictors of clinical and nerve conduction velocity (NCV) detected peripheral neuropathy in 50 children and adolescents with type 1 diabetes for a mean period of around $5 \mathrm{y}$ [3]. They observed a high prevalence of NCV detected neuropathy (56\%) with a preponderance of motor involvement in the absence of clinically detected neuropathy. Expectedly poor glycemic control (HbA1C above 9\%) and longer duration of diabetes (above $5 \mathrm{y}$ ) predicted the development of the complication. The prevalence of neuropathy is similar to other groups with a corresponding duration of follow-up and NCV-based assessment $[4,5]$.

The high prevalence of peripheral neuropathy in type 1 diabetes observed in the study prompted the authors to recommend nerve conduction screening in all children and adolescents with type 1 diabetes. This would, however, pose a significant burden to the already overburdened children and adolescents with type 1 diabetes. The current International Society for Pediatric and Adolescent Diabetes (ISPAD) guidelines recommend clinical neuropathy

Anurag Bajpai

dr_anuragbajpai@yahoo.com

1 Department of Pediatric Endocrinology, Regency

Center for Diabetes, Endocrinology \& Research,

Regency City Clinic, Opposite PPN Market, Kanpur,

Uttar Pradesh 208001, India

2 Fortis Memorial Research Institute, Gurgaon, Haryana, India screening (reflexes, temperature, monofilament) in children and adolescents with type 1 diabetes while restricting nerve conduction studies for severe cases or research purposes [6]. The substantially higher prevalence of neuropathy in subjects with a longer duration of diabetes and poor glycemic control in the study suggests restriction of the study to the at-risk population. However, the study's retrospective nature is inadequate to address the issues related to the timing of initial and follow-up screening. A relatively small sample size, the inclusion of a high proportion of subjects with poor glycemic control (48\% with $\mathrm{HbA1C}$ above 9\%) and microvascular complications (30\% with albuminuria) along with the lack of a control group and information about confounding factors for neuropathy (B12 levels) further limit the generalizability of the study. Another important factor against universal NCV screening for diabetic neuropathy is the lack of an effective intervention to retard its progression in contradistinction to other complications (ACE inhibition for nephropathy; LASER treatment for retinopathy). The early identification of NCV detected neuropathy is thus not expected to translate into improved clinical outcomes. The study highlights the need for heightened awareness about neuropathy in type 1 diabetes; more evidence is required to justify universal NCV-based screening.

\section{Declarations}

Conflict of Interest None.

\section{References}

1. Kumar P, Krishna P, Reddy SC, Gurappa M, Aravind SR, Munichoodappa C. Incidence of type 1 diabetes mellitus and associated complications among children and young adults: results from Karnataka Diabetes Registry 1995-2008. J Indian Med Assoc. 2008;106:708-11. 
2. Ramachandran A, Snehalatha C, Sasikala R, Satyavani K, Vijay V. Vascular complications in young Asian Indian patients with type 1 diabetes mellitus. Diabetes Res Clin Pract. 2000;48:51-6.

3. Singh DP, Singh P, Sharma S, Aneja S, Seth A. Point prevalence of peripheral neuropathy in children and adolescents with type 1 diabetes mellitus. Indian J Pediatr. 2021. https://doi.org/10.1007/ s12098-021-03742-4.

4. Hajas G, Kissova V, Tirpakova A. A 10-yr follow-up study for detecting peripheral neuropathy in young patients with type 1 diabetes. Pediatr Diabetes. 2016;17:632-41.

5. Walter-Höliner I, Barbarini DS, Lütschg J, et al. High prevalence and incidence of diabetic peripheral neuropathy in children and adolescents with type 1 diabetes mellitus: results from a five-year prospective cohort study. Pediatr Neurol. 2018;80:51-60.

6. Donaghue KC, Marcovecchio ML, Wadwa RP, et al. ISPAD clinical practice consensus guidelines 2018: microvascular and macrovascular complications in children and adolescents. Pediatr Diabetes. 2018;19:262-74.

Publisher's Note Springer Nature remains neutral with regard to jurisdictional claims in published maps and institutional affiliations. 